\title{
Incorporating landscape heterogeneities in the spread of an epidemic in wildlife
}

\author{
L. Gerardo-Giorda, J. Keller, and A. Veneziani \\ BCAM - Basque Center for Applied Mathematics, Bilbao, Spain \\ lgerardo@bcamath.org \\ Department of Biostatistics, University of Washington, Seattle, WA, USA \\ kellerjp@uw.edu \\ Department of Mathematics and Computer Science, Emory University, Atlanta, GA, USA \\ ale@mathcs . emory . edu
}

\section{Introduction}

One of the main difficulties in the modeling and numerical simulation of the spread of an infectious disease in wildlife resides in properly taking into account the heterogeneities of the landscape. Forests, plains and mountains present different levels of hospitality, while large interstates, lakes and major waterways can provide strong natural barriers to the epidemic spread. A canonical approach has been to discretize both population and geography into geopolitical units and consider the movement of individuals from unit to unit [4]. This approach, however, does not well represent the biological realities of animal movement, since animals do not move at the scale of geopolitical units. We combine a standard SEI epidemiological model with a diffusion process to account for movement as a continuous process across a continuous region [1]. This results in a system of parabolic reaction-diffusion equations with nonlinear reaction term. Landscape heterogeneities are accounted for by including in the computational domain the significant geographical features of the area. We discretize the resulting model in time by an IMEX scheme and in space by finite elements. To show the effectiveness of the method, we present numerical simulation for rabies epidemics among raccoons in New York State.

\section{Continuous model for movement}

Let $x \in \Omega \subset \mathbb{R}^{2}$. The SEI model subdivides the population individuals at time $t$ into Susceptibles $S(t)$, Exposed $E(t)$, and Infectious $I(t)$. We consider as unknown the vector $\mathbf{u}(x, t)=[s(x, t), e(x, t), i(x, t)]^{T}$ of their densities in location $x$ at time $t$. Thus

$$
S(t)=\int_{\Omega} s(x, t) d x, \quad E(t)=\int_{\Omega} e(x, t) d x, \quad \text { and } \quad I(t)=\int_{\Omega} i(x, t) d x .
$$

In more general cases, a fourth group of Recovered individuals could be considered (SEIR models), but in our case study this class is empty, as rabies is lethal for raccoons. However, the extension of the following arguments to SEIR models is straightforward.

We model the spread of an epidemic in $\Omega$ by a vector generalization of the normalized Fischer's equation, a nonlinear system of reaction-diffusion equations of parabolic type

$$
\partial_{t} \mathbf{u}-\operatorname{div}(\boldsymbol{\nu} \nabla \mathbf{u})=(\mathrm{A}-\mathrm{B}(\mathbf{u})) \mathbf{u} \quad \text { in } \Omega \times[0, T],
$$

where 


$$
\boldsymbol{\nu}=\left[\begin{array}{ccc}
\boldsymbol{\nu}_{s} & 0 & 0 \\
0 & \boldsymbol{\nu}_{e} & 0 \\
0 & 0 & \boldsymbol{\nu}_{i}
\end{array}\right], \quad \mathrm{A}=\left[\begin{array}{ccc}
\alpha & \alpha & 0 \\
0 & -\sigma & 0 \\
0 & \sigma & -\phi
\end{array}\right], \quad \mathrm{B}(\mathbf{u})=\left[\begin{array}{ccc}
-\mu_{0} n & -\beta s & 0 \\
\beta i & -\mu_{0} n & 0 \\
0 & 0 & -\mu_{0} n
\end{array}\right]
$$

$n=s+e+i$ being the total population, $\alpha$ the reproduction rate, $\beta$ the infectiveness of a contact between a susceptible and an infectious individual, $1 / \sigma$ the latency period, and $1 / \phi$ the life expectancy of a rabid animal. We assume a density dependent natural mortality rate $\mu_{0} n$. System (1) is completed by a suitable initial value $\mathbf{u}^{0}$, and suitable boundary conditions on $\partial \Omega$. In general homogeneous Neumann boundary conditions are used to model an isolated environment, but other boundary conditions can be considered as well: an homogeneous Dirichlet boundary condition would model an hostile environment, while a Robin boundary condition would model a migratory dynamics.

\section{Modeling landscape heterogeneities}

We consider here two kinds of geographical heterogeneity in the region of interest, that are modeled in two different ways. Localized heterogeneities, such as the presence of major waterways and roads, locally drive the movement of the wild animals. Extended heterogeneities are particular features significantly affecting the dynamics of the infection in a whole region. This is the case, for instance, of lakes, forests and mountains.

\subsection{Localized heterogeneities}

The effect of localized heterogeneities on the population dynamics are included by locally modifying the diffusivity tensors. In each point of the river we identify a local frame of reference $\left(\mathbf{e}_{\tau}, \mathbf{e}_{n}\right)$, see Figure 1 (left). In a neighborhood $\Sigma$ of width $2 \varepsilon$ of the river the diffusivity is gradually decreased, from the regular value $\nu_{H}$ to a lower value $\nu_{L}$, as plotted in Figure 1 (right). By denoting with $\hat{y}$ is the distance from the river along $\mathbf{e}_{n}$, the compartmental diffusion tensor $\boldsymbol{\nu}_{k}(k=s, e, i)$ is first computed in the $\left(\mathbf{e}_{\tau}, \mathbf{e}_{n}\right)$ reference frame, then referred to the Cartesian global frame of reference, by means of a rotation matrix $\mathrm{R}$ aligning the local frame of reference to the global one

$$
\boldsymbol{\nu}_{k}=\mathrm{R} \hat{\boldsymbol{\nu}}_{k} \mathrm{R}^{T}, \quad \hat{\boldsymbol{\nu}}_{k}=\left[\begin{array}{cc}
\nu_{H} & 0 \\
0 & \nu_{H}-\left(\nu_{H}-\nu_{L}\right) \exp \left(1-\frac{\epsilon^{2}}{\epsilon^{2}-\hat{y}^{2}}\right)
\end{array}\right] .
$$

\subsection{Extended heterogeneities}

Strong heterogeneities between different areas naturally induce a partition of the computational domain into homogeneous subregions. To take into account different levels of hospitality, we act on both the carrying capacity of the region, and the diffusion coefficients. For instance, in a unhospital area, we both lower the carrying capacity to reduce the presence of animals at the disease-free equilibrium, and the diffusion coefficient to avoid massive migration towards such area.

\section{Finite dimensional approximation}

Let $\Delta t$ be a time step and let $t^{n}=n \Delta t$. System (1) is advanced in time by mixed implicit/explicit (IMEX) finite differences, where the nonlinear term is treated explicitly, while the principal part of the operator is treated implicitly. Space discretization is based 

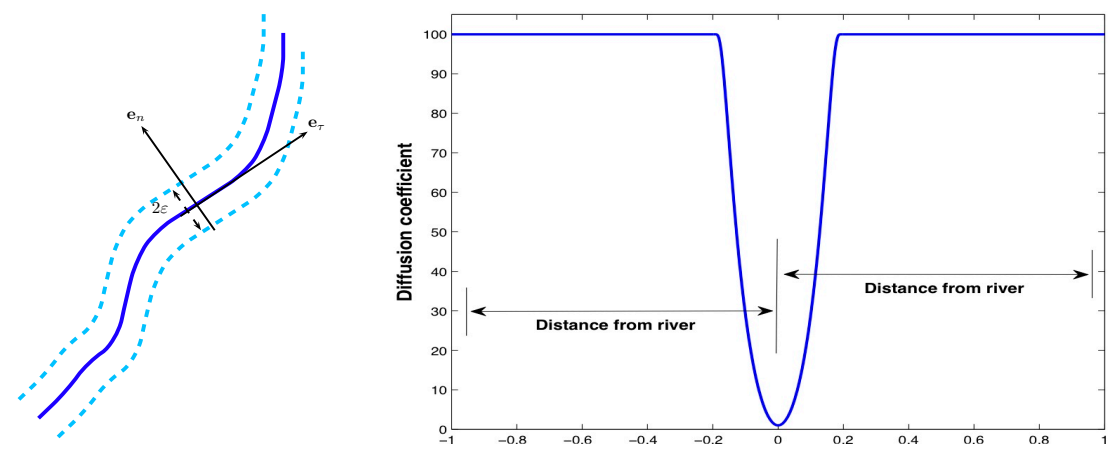

FIgURE 1. Left: $\left(\mathbf{e}_{\tau}, \mathbf{e}_{n}\right)$ reference frame along the river. Right: diffusion coefficient in the normal direction to the river, with $\nu_{H}=100, \nu_{L}=1$, and $\epsilon=0.2$. Figures from $[\mathbf{1}]$.

on finite elements (for an introduction to the method see e.g. [3]), since the latter are particularly suitable for complex geometries as real geographic regions can be. On a regular triangular mesh of the domain $\Omega$ with $N$ nodes, the unknown is the vector

$$
\mathbf{U}^{n}=\left[\left[\mathbf{s}_{l}\left(t^{n}\right)\right]_{l=1, \ldots, N},\left[\mathbf{e}_{l}\left(t^{n}\right)\right]_{l=1, \ldots, N},\left[\mathbf{i}_{l}\left(t^{n}\right)\right]_{l=1, \ldots, N}\right]
$$

of the nodal values at time $t^{n}$.

At each time step, we solve the algebraic system

$$
(\mathcal{M}+\Delta t \mathcal{A}) \mathbf{U}^{n}=\Delta t \mathcal{F}\left(\mathbf{U}^{n-1}\right)+\mathcal{M} \mathbf{U}^{n-1},
$$

where $\mathcal{M}$ and $\mathcal{A}$ are block-diagonal

$$
\mathcal{M}=\left[\begin{array}{ccc}
M & 0 & 0 \\
0 & M & 0 \\
0 & 0 & M
\end{array}\right], \quad \mathcal{A}=\left[\begin{array}{ccc}
A_{s} & 0 & 0 \\
0 & A_{e} & 0 \\
0 & 0 & A_{i}
\end{array}\right],
$$

where $M$ and $A_{k}(k=s, e, i)$ are the classical finite elements mass and stiffness matrices, and where $\mathcal{F}\left(\mathbf{U}^{n-1}\right)$ is obtained from the right hand side in (1) (see [1] for details).

\section{Numerical simulation}

For illustrative purpose we apply the method to the spread of a fictious epidemic of raccoon rabies in the state of New York. A simulation of the actual spread of rabies among raccoons in the area (1990-1996) can be found in [1], where the proposed method proves its effectiveness in qualitatively capturing the early dynamics of the epidemic.

\subsection{Computational domain}

A geographically detailed computational domain of the area of interest can be reconstructed from satellite images or recovered from other similar sources (GIS data, for instance), and suitably triangulated. The strongly heterogeneous regions are then identified directly on the mesh, while the localized heterogeneous effects are included by forcing mesh points in the areas of interest, such as major waterways and highways. We include in the geography the Hudson and Mohawk rivers as barriers, and the mountains in the northern part of the state as a reduced hospitality region. 


\subsection{Results}

The coefficients of the model used in the simulation refer to raccoon rabies. The epidemiological parameters $(\alpha, \beta, \sigma, \phi)$ are drawn from literature, while the mortality term $\mu_{0}$ is estimated indirectly to produce a disease-free equilibrium corresponding to the mean of reported densities for raccoons in the eastern US. We initiate an epidemic cluster in the vicinity of Oneonta (Otsego County), and we plot in Figure 2 the difference between the infectious density computed with the inclusion of the rivers and the one obtained without the presence of rivers at different times. The barrier effect is clearly appreciable.
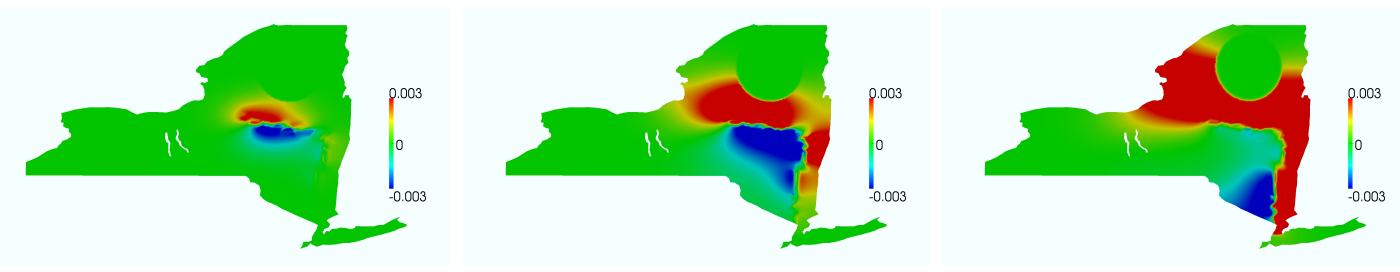

FIGURE 2. Effect of the presence of rivers and the reduced capacity region on the infectious density after 12, 24, and 36 months (left to right, [1]).

\subsection{Computational cost}

The algebraic system (3) does not present any significant difficulty from the numerical standpoint. Its $3 N \times 3 N$ matrix is symmetric, and the solution at each time step can be computed by a Conjugate Gradient (CG, $[\mathbf{3}]$ ) method, for which standard, very effective preconditioners are available.

\section{Conclusion}

The proposed space-continuous model features a double advantage. Geographic details are easily incorporated, and, at the same time, the overall computational cost is very competitive. As a matter of fact, the proposed model requires the tuning of a smaller number of parameters with respect to SEI models based on geopolitical units commonly used in the domain $([4])$. So far, the diffusion coefficients have been tuned empirically. To improve the knowledge of the model parameters, an estimation procedure of Bayesian type, based on the available observed data, is currently under investigation $([\mathbf{2}])$.

\section{References}

[1] J. Keller, L. Gerardo-Giorda and A. Veneziani (2012) Numerical simulation of a susceptible-exposedinfectious space-continuous model for the spread of rabies in raccoons across a realistic landscape. J. Biological Dynamics, DOI: 10.1080/17513758.2012.742578.

[2] G. Puggioni, L. Gerardo-Giorda, L.A. Waller and L.A. Real. Hierarchical Models for Parameter Estimation of SEIR Models with application to Raccoon Rabies spread (in preparation)

[3] A. Quarteroni and A. Valli. Numerical Approximation of Partial Differential Equations. SpringerVerlag, Berlin, 1994.

[4] L.A. Real and J.E. Childs. Spatial-temporal dynamics of rabies in ecological communities In S.K. Collinge and A.C. Ray, editors, Disease Ecology: Community structure and pathogen dynamics, pages 168-185. Oxford Univ. Press, 2006. 